\title{
EXISTENCE OF CHEBYSHEV CENTERS, BEST $n$-NETS AND BEST COMPACT APPROXIMANTS
}

BY

\author{
DAN AMIR, JAROSLAV MACH ${ }^{1}$ AND KLAUS SAATKAMP
}

\begin{abstract}
In this paper we investigate the existence and continuity of Chebyshev centers, best $n$-nets and best compact sets. Some of our positive results were obtained using the concept of quasi-uniform convexity. Furthermore, several examples of nonexistence are given, e.g., a sublattice $M$ of $C[0,1]$, and a bounded subset $B \subset M$ is constructed which has no Chebyshev center, no best $n$-net and not best compact set approximant.
\end{abstract}

1. Introduction. It is a familiar situation when one wants to replace a set $A$ of given data by another one $C$ which is more condensed or of a simpler type. If we have some metric on our data space $X$ it is natural to try to replace the set $A$ belonging to the class $\mathscr{Q}$ of admissible given data sets by a set $C$ belonging to the class $\mathcal{C}$ of admissible "approximating" sets such that the elements of $A$ will be as close to $C$ as possible. More explicitly, if we denote, in the metric space $(X, d), B(C, r)=$ $\{x ; d(x, C) \leqslant r\}$ (the closed $r$-neighbourhood of $C$ ), $r(C, A)=\min \{r ; A \subset B(C, r)\}$ and $r_{\mathcal{C}}(A)=\inf \{r(C, A) ; C \in \mathcal{C}\}$, then we are looking for $C_{0} \in \mathcal{C}$ with $r\left(C_{0}, A\right)=$ $r_{\mathcal{Q}}(A)$. We denote by $Z_{\mathcal{C}}(A)$ the set of all such $C_{0} \in \mathcal{C}$.

We shall deal here with the following typical classes of subsets of a Banach space $X$ : $\mathscr{G}_{3}(V)$ - the closed and bounded subsets of a closed $V \subset X ; \mathscr{K}(V)$ - the compact subsets of $V$; and $\mathscr{F}_{n}(V)$ - the class of sets consisting of at most $n$ points of $V$. $\mathscr{B}, \mathscr{K}, \mathscr{F}_{n}$ will stand, respectively, for $\mathscr{B}(X), \mathcal{K}(X)$ and $\mathscr{F}_{n}(X), V$ will stand for $\mathscr{F}_{1}(V)$, and $r(A), Z(A)$ - for $r_{X}(A), Z_{X}(A), r(A)$ is called the Chebyshev radius of $A$ and $Z(A)$ the Chebyshev center set of $A$. Similarly, $r_{V}(A)$ is called the relative Chebyshev radius of $A$ with respect to $V$ and $Z_{V}(A)$ the set of relative Chebyshev centers for $A$ in $V$.

The case $\Theta=X, \varrho=V$ is the classical best approximation problem $-r_{V}(x)$ is the distance $d(x, V)$ and $Z_{V}$ is the metric projection operator $P_{V}$ corresponding to $x \in X$ the set of nearest points to $x$ in $V$. (On the other hand, the general case can be reduced to the classical best approximation problem if we endow $\mathscr{B}(X)$ with the

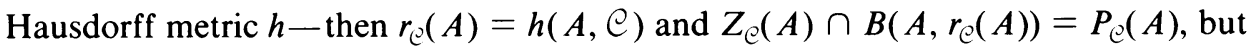
of course the concrete problem does not become simpler.)

Received by the editors September 26, 1980 and, in revised form, March 23, 1981.

1980 Mathematics Subject Classification. Primary 41A65, 41A28, 46B20, 46B25.

${ }^{1}$ The work of the first (resp. second) author was partly performed when he was a guest of the Sonderforschungsbereich 72 at the Institut für Angewandte Mathematik der Universität Bonn (resp. of the Department of Mathematical Sciences, Tel Aviv University) for a period of two weeks in July 1979 (resp. April-May 1980). 
Elements of $Z_{\widetilde{F}_{n}}(A)$ are called best $n$-nets of $A$.

$r_{\Im h}(A)$ is easily seen to be the Kuratowski measure of noncompactness of $A$, $\alpha(A)=\inf \left\{r \geqslant 0 ; \exists n, F \in \mathscr{F}_{n}\right.$ with $\left.r(F, A) \leqslant r\right\}$.

The concept of Chebyshev centers and best $n$-nets was introduced by Garkavi [8] and most of the basic results are due to him. Concerning existence problems, he observed the following two existence principles.

PROPOSITION A. If $V$ carries another topology $\tau$ such that $\underset{\alpha}{\stackrel{\tau}{\rightarrow} y \Rightarrow d(x, y)}$ $\leqslant \lim d\left(x, y_{\alpha}\right) \forall x \in X$, then a $\tau$-accumulation point of a "minimizing sequence" for the Chebyshev radius is necessarily a Chebyshev center. In particular, if $X$ is a dual normed space, then for every $w^{*}$-closed $V \subset X, Z_{V}(A) \neq \varnothing \forall A \in \mathscr{B}(X)$.

The same argument applies also to best $n$-nets.

Proposition B. If $P$ is a contractive projection of $X$ onto $X_{0}$ and $P \mathcal{C} \subset \mathcal{C}$, then $P Z_{\mathrm{E}}(A) \subset Z_{P \mathrm{E}}(A) \forall A \in \mathscr{B}\left(X_{0}\right)$. In particular, if $X$ is Banach space admitting a norm-one-projection from its bidual (e.g. $X=L_{1}(\mu)$ for any $\sigma$-finite $\mu$ ), then $Z_{\bar{s}_{n}}(A) \neq$ $\varnothing \forall A \in G(X)$.

Approximation of bounded sets by compact sets was investigated in [23], in connection with questions of best approximating bounded linear operators by compact ones. M. Feder [7] constructed a bounded subset $A$ of $L_{1}[0,1]$ which admits no best compact set approximant. This shows that the analogue of Proposition B does not hold for the class $\mathcal{K}$. On the other hand, it follows from the results of Fakhoury [6] and Mach and Ward [23] that every bounded subset of $l_{1}$ has a best compact set approximant.

The existence of Chebyshev centers for bounded subsets is established, by Propositions A and $\mathrm{B}$, for the classical spaces $L_{p}(\mu), 1 \leqslant p<\infty$. For the other classical space, $C(\Omega)$ ( $\Omega$ compact Hausdorff), the existence of Chebyshev centers for bounded sets was established by Kadec and Zamyatin [12]. There are several ways of proving this:

We first observe that the problem of finding $Z(A)$ in $C(\Omega)$ reduces to the problem of finding $Z_{C(\Omega)}\{(\inf A, \sup A)\}$ in $m(\Omega)$, and this is equivalent to finding $x \in C(\Omega)$ satisfying sup $A-r(A) \leqslant x \leqslant \inf A+r(A)$, which can be guaranteed by the HahnTong interposition theorem [27, p. 100] or by Michael's selection theorem. Another way is a "successive approximation" argument, which will be exploited in $\$ 2$.

Garkavi [8] also gave the first example of nonexistence of Chebyshev centers: He showed that if $X$ is the nonproximinal hyperplane $\left\{x \in C[-1,1] ; \int_{-1}^{0} x(t) d t=\right.$ $\left.\int_{0}^{1} x(t) d t\right\}$ of $C[-1,1]$, then $Z(A)=\varnothing$ for some $A \in \mathscr{F}_{3}$. Garkavi showed later [9] that if $X$ is a finite-codimensional subspace of $C(\Omega)$, then $Z_{X}(A) \neq \varnothing \forall A \in$ $\mathcal{H}(C(\Omega))$ iff $X$ is proximinal. Garkavi and Zamyatin [10] showed that for such a proximinal $X, Z_{X}(A) \neq \varnothing \forall A \in \emptyset(C(\Omega))$ iff the support of every $\mu \in X^{\perp}$ is "extremally disconnected with respect to $\Omega$ " (i.e. $\bar{G} \cap \operatorname{spt} \mu$ is open in spt $\mu$ for every open $G \subset \Omega$ ). Some remarks concerning these examples are made in $\S 3$.

It is now natural to investigate the existence of Chebyshev centers for bounded and compact sets in the "semiclassical" Banach spaces. As such can be considered 
the Lindenstrauss spaces (preduals of $L_{1}$-spaces) and the spaces $C(\Omega, X), L_{p}(\mu, X)$ of vector-valued functions, where $X$ is "classical". The case of Lindenstrauss spaces is studied in $\S 4$, and the case of vector-valued functions in $\$ 5$.

2. Quasi-uniform convexity and the successive approximation argument. We say that the normed space $X$ is quasi-uniformly convex (q.u.c.) with respect to its subspace $Y$ if $\forall \varepsilon>0 \exists 0<\tilde{\delta}<1$ such that $\forall y \in Y \exists z \in Y$ with $\|z\| \leqslant \varepsilon$, and such that if $u \in X,\|u\| \leqslant 1$ and $\|u-y\| \leqslant 1-\tilde{\delta}$, then also $\|u-z\| \leqslant 1-\tilde{\delta}$. In the case $X=Y$ we say that $X$ is quasi-uniformly convex. $X$ is said to be strictly convex with respect to $Y$ if $y-z \in Y,\|y\|=\|z\|=\left\|\frac{1}{2}(y+z)\right\| \rightarrow y=z$, and uniformly convex with respect to $Y$ if $\forall \varepsilon \exists \delta$ such that $y-z \in Y,\|y\|=\|z\|=1,\|y-z\| \geqslant \varepsilon \Rightarrow$ $\left\|\frac{1}{2}(y+z)\right\| \leqslant 1-\delta[3]$.

The concept of quasi-uniform convexity is due to Calder, Coleman and Harris [4].

2.1. LEMMA. If $\varepsilon<\frac{1}{2}$ and $\tilde{\delta}=\tilde{\delta}(\varepsilon)$ satisfies the q.u.c. condition, so does every $\eta \in(0, \tilde{\delta})$.

Proof. By homogeneity, $\eta$ satisfies the q.u.c. conditions with respect to $(1-\eta)$ $\cdot(1-\tilde{\delta})^{-1} \varepsilon \leqslant 1$. Therefore, given $y \in Y$ there is $u \in Y$ with $\|u\| \leqslant 1$ and $B(y, 1-\eta) \cap B(0,1) \subset B(u, 1-\eta)$. There is $z \in Y$ with $\|z\| \leqslant \varepsilon$ such that $B(u, 1-\tilde{\delta}) \cap B(0,1) \subset B(z, 1-\tilde{\delta})$, and then

$$
\begin{aligned}
u+(1-\tilde{\delta})(1-\eta)^{-1}[B(y, 1-\eta) \cap B(0,1)-u] & \subset B(u, 1-\tilde{\delta}) \cap B(0,1) \\
& \subset B(z, 1-\tilde{\delta}) .
\end{aligned}
$$

Let $w=u+(1-\eta)(1-\tilde{\delta})^{-1}(z-u)$. Then $B(y, 1-\eta) \cap B(0,1) \subset B(w, 1-\eta)$, and since $z \in[u, w]$, also $B(y, 1-\eta) \cap B(0,1) \subset B(z, 1-\eta)$.

2.2. Proposition. The following are equivalent:

(a) $X$ is uniformly convex with respect to $Y$.

(b) $X$ is q.u.c. with respect to $Y$ and strictly convex with respect to $Y$.

(c) $X$ is q.u.c. with respect to $Y$ and we can take $z \in[0, y]$.

Proof. If $X$ is uniformly convex with respect to $Y$ then it is clearly strictly convex with respect to $Y$. Given $y \in Y, \varepsilon>0$, let

$$
z= \begin{cases}y & \text { if }\|y\| \leqslant \varepsilon \\ \varepsilon y /\|y\| & \text { if }\|y\| \geqslant \varepsilon\end{cases}
$$

If $\|u\| \leqslant 1$ and $\|u-y\| \leqslant 1-\delta(\varepsilon)$, then $\left\|u-\frac{1}{2} y\right\| \leqslant 1-\frac{1}{2} \delta(\|y\|)$. If $\|y\| \geqslant \varepsilon$ then

$$
\begin{aligned}
\|u-z\| & =\left\|u-\frac{\varepsilon}{\|y\|} y\right\| \leqslant \frac{2 \varepsilon}{\|y\|}\left\|u-\frac{y}{2}\right\|+\left(1-\frac{2 \varepsilon}{\|y\|}\right)\|u\| \\
& \leqslant \frac{2 \varepsilon}{\|y\|}\left(1-\frac{1}{2} \delta(\|y\|)\right)+1-\frac{2 \varepsilon}{\|y\|}=1-\frac{\varepsilon}{\|y\|} \delta(\|y\|) \leqslant 1-\delta(\varepsilon)
\end{aligned}
$$

(since $\delta(t) / t$ increases with $t$ ). If $\|y\| \leqslant \varepsilon$ then $\|u-z\|=\|u-y\| \leqslant 1-\delta(\varepsilon)$. Thus $\tilde{\delta}(\varepsilon)=\delta(\varepsilon)$. Thus (a) $\Rightarrow(\mathrm{b}),(\mathrm{c})$. 
If $X$ is q.u.c. with respect to $Y$ and we can always take $z \in[0, y]$, let $d=\delta(\varepsilon / 3)$. If $\|u\|=\|v\|=1,\|u-v\| \geqslant \varepsilon$ and $u-v \in Y$, there is $z \in[v,(1-d) u]$ with $\|z-v\| \leqslant \varepsilon / 3$ and $\|z\| \leqslant 1-d$. Let $w=(1-d)(2-d)^{-1}(u+v)$. Then $w \in$ $[(1-d) u, v]$ and

$$
\|w-v\|=(2-d)^{-1}\|(1-d) u-v\|>\frac{1}{2}\|(1-d) u-v\| \geqslant \varepsilon / 3 ;
$$

$w \in[(1-d) u, z]$ and $\|w\| \leqslant 1-d$. Therefore

$$
\left\|\frac{1}{2}(u+v)\right\|=\frac{1}{2}(2-d)(1-d)^{-1}\|w\| \leqslant 1-\frac{1}{2} d,
$$

so that $\delta(\varepsilon) \geqslant \frac{1}{2} \tilde{\delta}(\varepsilon / 3)$. Thus (c) $\Rightarrow(\mathrm{a})$.

Finally, if $X$ is q.u.c. with respect to $Y$ and strictly convex with respect to $Y$, and $\|u\|=\|v\|=1$ satisfy $u-v \in Y,\|u-v\| \geqslant 4 \varepsilon$ and if $\left\|\frac{1}{2}(u+v)\right\|=1-d$ where $d \leqslant \tilde{\delta}(\varepsilon)$, then by Lemma 2.1 there is $z \in Y$ with $\|z\| \leqslant \varepsilon$ and $B\left(\frac{1}{2}(u-v), 1-d\right) \cap$ $B(0,1) \subset B(z, 1-d)$. Since $\|u-(u-v) / 2\|=\|-v-(u-v) / 2\|=1-d$, we have $\|u-z\| \leqslant 1-d,\|v+z\| \leqslant 1-d$ and $\left\|\frac{1}{2}(u+v)\right\| \leqslant 1-d$. Equality implies, by strict convexity, $u-z=v+z$, i.e. $z=\frac{1}{2}(u-v)$ and we get the contradiction $\varepsilon>\|z\| \geqslant 2 \varepsilon$. Therefore $\delta(4 \varepsilon) \geqslant \tilde{\delta}(\varepsilon)$.

Remark. Proposition 2.2 is, essentially, due to Calder, Coleman and Harris [4].

Examples of nonstrictly convex spaces which are q.u.c. are the $C(\Omega)$ spaces and some of their subspaces, as shown in Proposition 4.4.

2.3. Proposition. If $X$ is q.u.c. and $Y$ is the range of a norm-one projection $P$ in $X$, then $Y$ is also q.u.c. and $\tilde{\delta}_{Y}(\varepsilon) \geqslant \tilde{\delta}_{X}(\varepsilon)$.

Proof. Given $y \in Y, \varepsilon>0$, let $\|z\| \leqslant \varepsilon, z \in X$ satisfy

$$
B\left(z, 1-\tilde{\delta}_{X}(\varepsilon)\right) \supset B\left(y, 1-\tilde{\delta}_{X}(\varepsilon)\right) \cap B(0,1) .
$$

Then $\|P z\| \leqslant \varepsilon$ and

$$
B\left(P z, 1-\tilde{\delta}_{X}(\varepsilon)\right) \supset B\left(y, 1-\tilde{\delta}_{X}(\varepsilon)\right) \cap B(0,1) \cap Y .
$$

2.4. Proposition. If a Banach space $X$ is q.u.c. with respect to a closed subspace $Y$, then:

(a) Every bounded $A \subset X$ has a nonempty Chebyshev center set in $Y, Z_{Y}(A)$, and $A \rightarrow Z_{Y}(A)$ is uniformly continuous on $\left\{A ; r_{Y}(A) \leqslant R\right\}$ for every $R>0$.

(b) Every bounded set $A \subset X$ has a nonempty set of best compact set approximants in $Y, Z_{K_{(Y)}}(A)$, and the mapping $A \rightarrow Z_{K_{(Y}(Y)}(A)$ is uniformly continuous on $\left\{A ; r_{Y}(A)\right.$ $\leqslant R\}$ for every $R>0$.

Proof. (a) For a $\gamma>0$ let $y_{n}$ satisfy

$$
r\left(y_{n}, A\right) \leqslant \frac{r_{Y}(A)}{\prod_{k=n}^{\infty}\left(1-\tilde{\delta}\left(2^{-k} \gamma\right)\right)}
$$

(since $\tilde{\delta}(\eta) \leqslant \eta$, the product converges). Let $z_{1}=y_{1} \cdot z_{n+1} \in Y$ can be chosen by the q.u.c. property so that

$$
\left\|z_{n+1}-z_{n}\right\| \leqslant 2^{-n} \gamma r\left(y_{n}, A\right) \quad \text { and } \quad r\left(z_{n+1}, A\right) \leqslant \frac{r_{Y}(A)}{\prod_{k=n+1}^{\infty}\left(1-\tilde{\delta}\left(2^{-k} \gamma\right)\right)} .
$$


The sequence $\left(z_{n}\right)$ is Cauchy and converges to a Chebyshev center $z \in Z_{Y}(A)$, and $\left\|z-y_{1}\right\| \leqslant \gamma r\left(y_{1}, A\right)$.

To prove the uniform continuity of $Z_{Y}$ let $\varepsilon>0$ be given. Let $\gamma=\varepsilon / 2 R$, and $h\left(A, A^{\prime}\right)=\eta r_{Y}(A)$ with $\eta \leqslant \frac{1}{2} \tilde{\delta}(\gamma / 2)$. Then $\left|r_{Y}(A)-r_{Y}\left(A^{\prime}\right)\right| \leqslant \eta r_{Y}(A)$ and for $z \in Z_{Y}(A), r\left(z, A^{\prime}\right) \leqslant(1+\eta) r_{Y}(A)$. Furthermore,

$$
(1+\eta) r_{Y}(A) \leqslant \frac{(1-\eta) r_{Y}(A)}{1-\tilde{\delta}(\gamma / 2)} \leqslant \frac{r_{Y}\left(A^{\prime}\right)}{\prod_{k=1}^{\infty}\left(1-\tilde{\delta}\left(2^{-k} \gamma\right)\right)}
$$

and, therefore, by the first part of the proof, there is $z^{\prime} \in Z_{Y}\left(A^{\prime}\right)$ satisfying $\left\|z-z^{\prime}\right\| \leqslant \gamma(1+\eta) r_{Y}(A)$. Thus $h\left(Z_{Y}(A), Z_{Y}\left(A^{\prime}\right)\right) \leqslant \varepsilon$.

(b) Let $F_{n} \in \mathscr{F}_{n}(Y)$ satisfy

$$
r\left(F_{n}, A\right) \leqslant \frac{r_{\mathscr{F}_{n}(Y)}(A)}{\prod_{k=n}\left(1-\tilde{\delta}\left(2^{-k} \varepsilon\right)\right)}
$$

Let $G_{1}=F_{1}$. Having $G_{n-1}$, replacing each point $x$ of $G_{n-1}$ by an $n$-tuple of points in $B\left(x, \varepsilon / 2^{n}\right) \cap Y$, we get $G_{n}$ of cardinality $n$ ! satisfying $r\left(G_{n}, A\right) \leqslant r\left(F_{n}, A\right)$. $G=\overline{\cup G_{n}}$ is compact and $r(G, A)=r_{\mathcal{K}(Y)}(A)$. The proof of the uniform continuity is analogous to part (a).

2.5. EXAMPLE. $A$ 3-dimensional space which is not quasi-uniformly convex: Let $B$ be the symmetric convex hull of the circle $\left\{\cos t e_{1}+\sin t e_{2} ; 0 \leqslant t \leqslant 2 \pi\right\}$ and the segment $\left\{s e_{1}+e_{3} ;-1 \leqslant s \leqslant 1\right\}$, and $\|\cdot\|$ the norm whose unit ball is $B$, i.e.

$$
\|(x, y, z)\|= \begin{cases}\sqrt{y^{2}+(|x|-|z|)^{2}}+|z| & \text { if }|x| \geqslant|z|, \\ |y|+|z| & \text { if }|x| \leqslant|z| .\end{cases}
$$

Since $Z\left(-e_{1}, e_{1}\right)=\left[-e_{3}, e_{3}\right]$ and $Z\left(-e_{1}-\eta e_{2}, e_{1}+\eta e_{2}\right)=0$, it is clear that $A \rightarrow$ $Z(A)$ is not continuous, so that the space is not quasi-uniformly convex. Indeed, let

$$
\begin{aligned}
& x=(1-\eta) e_{3}, \\
& u=\left(1-\frac{1}{2} \eta\right) e_{1}+\frac{1}{2} \sqrt{3} e_{2}+(1-\eta) e_{3}, \\
& v=-\left(1-\frac{1}{2} \eta\right) e_{1}-\frac{1}{2} \sqrt{3} e_{2}+(1-\eta) e_{3} . \text { Then } \quad r(x,[u, v])=\left(1-\eta+\eta^{2}\right)^{1 / 2}<
\end{aligned}
$$
$r(z,[u, v])$ for every $z$ with $\|z\|<1$. This shows that $\tilde{\delta}(\varepsilon)=0 \forall \varepsilon<1$.

2.6. Proposition. If $(\Omega, \mu)$ contains $2 n+1$ disjoint sets of finite positive measure, then $\tilde{\delta}_{L_{1}(\mu)}(\varepsilon) \leqslant \varepsilon /(2 n+1) \forall \varepsilon<1$.

Proof. Let $A_{1}, \ldots, A_{2 n+1}$ be disjoint of finite positive measure. Let

$$
x=\frac{1}{2 n+1} \sum_{i=1}^{2 n+1} \frac{A_{i}}{\mu\left(A_{i}\right)}
$$

and suppose $z$ satisfies the q.u.c. condition for $x, \varepsilon$ and $\varepsilon /(2 n+1)$. Denote $\alpha_{i}=$ $\int_{A_{i}}\left|z_{i}\right| d \mu$. We may assume $\alpha_{1} \geqslant \alpha_{2} \geqslant \cdots \geqslant \alpha_{2 n+1}$. Let

$$
u=\frac{1}{n+1} \sum_{i=n+1}^{2 n+1} \frac{\chi A_{i}}{\mu\left(A_{i}\right)} \text {. }
$$


Clearly $\|u\|=1$,

$$
\|u-x\|=\sum_{i=1}^{n} \frac{1}{2 n+1}+\sum_{i=n+1}^{2 n+1}\left(\frac{1}{n+1}-\frac{1}{2 n+1}\right)=1-\frac{1}{2 n+1},
$$

while

$$
\begin{aligned}
\|u-z\| & \geqslant \sum_{i=1}^{n} \alpha_{i}+\sum_{i=n+1}^{2 n+1} \frac{1}{n+1}-\sum_{i=n+1}^{2 n+1} \alpha_{i}=1+2 \sum_{i=1}^{n} \alpha_{i}-\sum_{i=1}^{2 n+1} \alpha_{i} \\
& \geqslant 1+\left(\frac{2 n}{2 n+1}-1\right) \sum_{i=1}^{2 n+1} \alpha_{i} \geqslant 1-\frac{1}{2 n+1} \varepsilon .
\end{aligned}
$$

2.7. COROLlaRY. Infinite-dimensional $L_{1}(\mu)$-spaces are not quasi-uniformly convex.

Remark. The case $L_{1}[0,1]$ follows also from Proposition 2.4 and results of Feder [7]. In this case we have the stronger result which will be used in $§ 5$ :

2.8. Proposition. In $L_{1}[0,1]$, if $x=1$, no $\|z\|<1$ and $d \in(0,1)$ satisfy

$$
B(x, 1-d) \cap B(0,1) \subset B(z, 1-d) \text {. }
$$

Proof. Take $A$ with $\mu(A)=(1-d) / 2$ and $\int_{A}|z| d \mu$ maximal, and let $u=$ $2\left(1-\chi_{A}\right) /(1+d)$. Then $\|u\|=1$,

$$
\|u-x\|=\frac{1-d}{1+d} \frac{1+d}{2}+\frac{1-d}{2}=1-d
$$

while

$$
\begin{aligned}
\|u-z\| & \geqslant \int_{A}|z| d \mu+\int_{A^{c}} u d \mu-\int_{A} c|z| d \mu=2 \int_{A}|z| d \mu-\|z\|+1 \\
& \geqslant 1-\|z\|+2 \cdot \frac{1}{2}(1-d)\|z\|=1-\|z\| d .
\end{aligned}
$$

REMARK. The q.u.c. property is the simultaneous approximation version of a simpler property which was studied, in several variants, by Holmes [11], Lau [14] and others:

Call a subspace $Y$ of $X$ " $U$-proximinal" if $\forall \varepsilon>0 \exists \delta>0$ such that

$$
B(0,1) \cap(Y+B(0,1-\delta)) \subset(B(0, \varepsilon) \cap Y)+B(0,1-\delta),
$$

i.e. such that if $\|u\| \leqslant 1, y \in Y,\|u-y\| \leqslant 1-\delta$, then $\|u-z\| \leqslant 1-\delta$ for some $z \in Y,\|z\| \leqslant \varepsilon$.

This property is weaker than q.u.c. and is enjoyed, e.g., by "semi- $M$-ideals" $Y$ in any Banach space $X$ (with $\delta=\varepsilon$ ).

$Y$ is called "locally $U$-proximinal" in $X$ if it is $U$-proximinal in $\operatorname{span}\{Y, x\}$ $\forall x \in X$.

Local $U$-proximinality implies proximinality in a way analogous to Proposition 2.4 , and in the $U$-proximinal case the metric projection is continuous.

3. Nonexistence of centers and best compact approximants in hyperplanes of $C(\Omega)$ and isomorphs of $C(\Omega)$. In the particular case $\Omega=[a, b]$ (or, more generally, any metric compact), the Garkavi-Zamyatin result shows that the finite codimensional 
subspace $X=\left[\mu_{1}, \ldots, \mu_{n}\right]_{\perp}$ of $C(\Omega)$ admits relative centers for bounded sets in $C(\Omega)$ iff each of the annihilating measures has a finite support [31]. In fact, if this fails we get more nonexistence results.

3.1. Example. Let $X=\left\{x \in C[0,1] ; \int_{0}^{1} x(t) d t=\frac{1}{4}\right\}, \quad A=\{x \in X ;|x(t)|$ $\left.\leqslant \frac{1}{2} \chi_{[0,1 / 2]}(t)+\chi_{(1 / 2,1]}(t)\right\}$. Then $A$ has no best $n$-net for any $n \geqslant 1$ and no best compact approximant in $X$.

Proof. For any $n \in \mathbf{N}$,

$$
\begin{aligned}
x_{n}(t)= & \frac{1}{2} \chi_{[0,1 / 2-1 / 2 n]}(t)+\frac{2 n^{2}(1-2 t)+1}{2(2 n+1)} \chi_{(1 / 2-1 / 2 n, 1 / 2]}(t) \\
& +(1 / 2(2 n+1)) \chi_{[1 / 2,1]}(t)
\end{aligned}
$$

satisfies $x_{n} \in X$ and $r\left(x_{n}, A\right)=1+1 / 2(2 n+1) \rightarrow 1$.

Suppose now $K \subset X$ is compact; then it is equicontinuous and therefore there is $0<\delta<\frac{1}{10}$ such that $|s-t|<\delta, y \in K$, implies $|y(s)-y(t)|<\frac{1}{10}$. Let $x \in A$ oscillate between the upper and lower bounds faster than $\eta$, where $0<\eta<\frac{1}{100}$ is such that $|s-t|<\eta, y \in K$, implies $|y(s)-y(t)|<\delta / 10$, and suppose $y \in K$ satisfies $\|x-y\| \leqslant 1$. Then $y$ satisfies $|y| \leqslant \delta / 10$ in $\left[\frac{1}{2}, 1\right]$. We also must have $y \leqslant \frac{1}{2}+\delta / 10$ on $\left[0, \frac{1}{2}\right]$; hence

$$
\int_{0}^{s} y(t) d t \leqslant s\left(\frac{1}{2}+\frac{1}{10} \delta\right) \quad \forall s \in\left[0, \frac{1}{2}\right]
$$

and for some $t \in\left[s, \frac{1}{2}\right]$ we must have

$$
y(t) \geqslant\left(\frac{1}{4}-\frac{1}{20} \delta-s\left(\frac{1}{2}+\frac{1}{10} \delta\right)\right) /\left(\frac{1}{2}-s\right) .
$$

For this $t$ we have

$$
y(t)-y\left(\frac{1}{2}\right) \geqslant \frac{1}{2}-\left(\frac{\delta / 10+2 s \delta / 10}{1-2 s}+\frac{\delta}{10}\right)=\frac{1}{2}-\frac{2 \delta / 10}{1-2 s} .
$$

Taking $s=(1-\delta) / 2$, we get $y(t)-y\left(\frac{1}{2}\right)=\frac{1}{2}-\frac{2}{10}>\frac{1}{10}$, which contradicts the choice of $\delta$. Thus $r(K, A)>1 \forall K \in \mathscr{K}$.

REMARKs. The fact that the sets of type of $A$ fail to have centers in $X$ was observed by Smith and Ward [28].

The next example is similar, only that this time we have nonexistence in a strictly convex isomorph of $C[-1,1]$.

3.2. EXAMPLE. Let $X$ be $C[-1,1]$ with the norm $\|x\|=\|x\|_{\infty}+\|x\|_{2}, A=\{x \in$ $C[-1,1] ;-2 \leqslant x \leqslant 0$ on $[-1,0), 0 \leqslant x \leqslant 2$ on $(0,1]\}$. Then $A$ has no Chebyshev center in $X$.

Proof. For any $n \in \mathbf{N}$,

$$
x_{n}=\chi_{[-1,-1 / n)}+n t \chi_{[-1 / n, 1 / n)}+\chi_{[1 / n, 1]}
$$

satisfies

$$
\begin{aligned}
r\left(x_{n}, A\right) & =2+\left[2\left(1-\frac{1}{n}\right)+2 \int_{0}^{1 / n}(2-n t)^{2} d t\right]^{1 / 2} \\
& =2+\sqrt{2+8 / 3 n} \rightarrow 2+\sqrt{2} .
\end{aligned}
$$


Suppose $z \in Z(A)$. Let ${ }^{*}$ denote the involution $x^{*}(t)=-x(-t)$ in $X$, and let $\hat{x}=\frac{1}{2}\left(x+x^{*}\right)$. Since $A=A^{*}=\hat{A}$, we have $r(\hat{y}, A)=r(y, A) \forall y$, so that we may assume that the Chebyshev center $z$ is an odd function. Define

$$
f(t)=2-\frac{1}{6} t+\sqrt{2}\left[\frac{1}{2} t\left(\frac{7}{4}\right)^{2}+\left(1-\frac{3}{2} t\right)\left(1+\frac{1}{6} t\right)^{2}\right]^{1 / 2} .
$$

Let $0<\delta<1$ be such that $|s-t| \leqslant \delta \Rightarrow|z(s)-z(t)|<\frac{1}{4}$ and such that $f(\delta)>2+$ $\sqrt{2}$. Further, let $0<\eta<\delta / 2$ be such that $|s-t| \leqslant \eta \Rightarrow|z(s)-z(t)|<\delta / 6$.

Let $x$ be odd and satisfy $\mu\{t \in[0,1] ;|x(t)-1| \neq 1\}<\delta / 2, x(t)=2$ if $t \in[\eta, 1]$ and $z(t) \leqslant 1-\delta / 6$, and $x(t)=0$ if $t \in[\eta, 1]$ and $z(t) \geqslant 1+\delta / 6$. Then

$$
\begin{aligned}
\|x-z\| & \geqslant|(x-z)(\eta)|+\sqrt{2}\left(\int_{0}^{\delta}|x(t)-z(t)|^{2} d t+\int_{\delta}^{1}|x(t)-z(t)|^{2} d t\right)^{1 / 2} \\
& \geqslant f(\delta)>2+\sqrt{2} .
\end{aligned}
$$

4. Lindenstrauss spaces. Recall the Lindenstrauss-Wulbert diagram of pre- $L^{1}$ spaces:

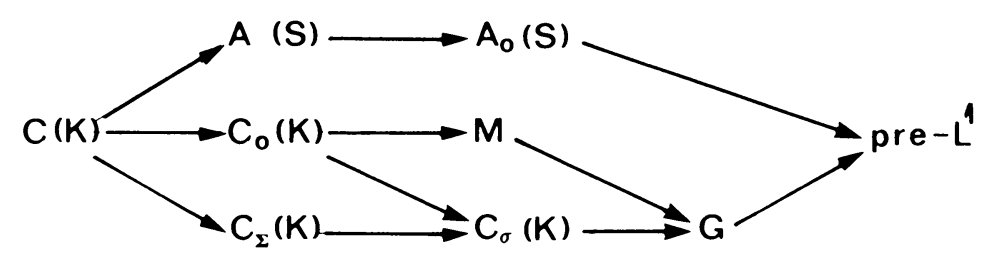

We want to find out in which of these spaces Chebyshev centers and best compact set approximants exist.

4.1. Proposition. If compact sets in the Banach space $X$ have relative Chebyshev centers in the subspace $Y$, then they also have best $n$-nets in $Y$ for all $n \in \mathbf{N}$.

Proof. Let $K \in \mathscr{K}(X)$, and let $\hat{K}$ be its canonical embedding in $X^{* *}$. $K$ has a best $n$-net in $Y^{\perp \perp}, y_{1}, \ldots, y_{n}$ (by Proposition B). Let $r=r_{\overline{v F}_{n}(Y)}(K), \hat{r}=r_{\bar{v}_{n}\left(Y^{\perp \perp}\right.},(\hat{K})$, $K_{i}=K \cap B\left(y_{i}, \hat{r}\right), z_{i} \in Z_{Y}\left(K_{i}\right), i=1, \ldots, n$. By a result of Lima [16, Corollary 1.3], it follows that $r_{Y}(E)=r_{Y^{\perp \perp}}(\hat{E})$ for all $E \in \mathcal{K}(X)$, hence $r\left(z_{i}, K_{i}\right)=r_{Y^{\perp \perp}}\left(\hat{K}_{i}\right) \leqslant \hat{r}$ $\leqslant r$, and $\left\{z_{1}, \ldots, z_{n}\right\} \in Z_{\bar{F}_{n}(Y)}(K)$.

Remarks. (1) The converse is obvious-if $Z_{\bar{\sigma}_{n+1}(Y)}(K) \neq \varnothing$ for all $K \in \mathcal{K}(X)$, then also $Z_{\mathscr{F}_{n}(Y)}(K) \neq \varnothing$ for all $K \in \mathscr{K}(X)$-just add a remote point $y \in Y$ to $K$.

(2) If we assume $E$ only bounded, we may have $r_{Y}(E) \neq r_{Y^{\perp \perp}}(E)$, e.g., $X=Y=$ $C[-1,1], E=\{x,-1 \leqslant x \leqslant 0$ on $[-1,0], 0 \leqslant x \leqslant 1$ on $[0,1]\}$.

4.2. Proposition [19, Theorem 2.2]. If $Y$ is an $M$-ideal in an $L_{1}$-predual space $X$, then $Z_{Y}(K) \neq \varnothing$ for all $K \in \mathscr{K}(X)$.

4.3. COROLlaRY. If $Y$ is an $M$-ideal in an $L_{1}$-predual $X$, then $Z_{\bar{\sigma}_{n}(Y)}(K) \neq \varnothing$ for all $K \in \mathscr{K}(X)$.

4.4. Proposition. If $X$ is the linear sublattice of $C(\Omega)$ ( $\Omega$ compact Hausdorff) determined by the full set of conditions: $x\left(s_{\alpha}\right)=\lambda_{\alpha} x\left(t_{\alpha}\right), s_{\alpha}, t_{\alpha} \in \Omega, \lambda_{\alpha} \geqslant 0$, and if $\inf \left\{\lambda_{\alpha} ; \lambda_{\alpha}>0\right\}>\delta_{0}>0$, then $C(\Omega)$ is quasi-uniformly convex with respect to $X$, with $\tilde{\delta}(\varepsilon) \geqslant \delta_{0} \varepsilon$. 
Proof. Let $N=\{t \in \Omega ; x(t)=0 \forall x \in X\}$. If $t \notin N$, there is $y_{t} \in X$ with $\left\|y_{t}\right\| \leqslant 1$ and $y_{t}(t)>\delta_{0}$. Let $V_{t}=\left\{s ; y_{t}(s)>\delta_{0}\right\}$. Given $x \in X$, let $K_{0}=\left\{t ;|x(t)| \geqslant \delta_{0} \varepsilon\right\}$. By compactness there are $t_{1}, \ldots, t_{m}$ such that $\bigcup_{i=1}^{m} V_{t_{i}} \supset K_{0}$. Let $y=\max _{1 \leqslant i \leqslant m} y_{t_{i}}$ and let $z=\varepsilon y \wedge x \vee(-\varepsilon y)$. Clearly $\|z\| \leqslant \varepsilon$, and if $u \in C(\Omega),\|u-x\| \leqslant 1-\delta_{0} \varepsilon$ and $\|u\| \leqslant 1$, consider for $t \in \Omega$ the three possible cases (we may assume $x(t) \geqslant 0$ ):

(i) $x(t) \leqslant \varepsilon y(t)$; then $|(u-z)(t)|=|u(t)-x(t)| \leqslant 1-\delta_{0} \varepsilon$.

(ii) $x(t) \geqslant \varepsilon y(t) \geqslant u(t)$; then $|(u-z)(t)| \leqslant|u(t)-x(t)| \leqslant 1-\delta_{0} \varepsilon$.

(iii) $x(t) \geqslant \varepsilon y(t), u(t) \geqslant \varepsilon y(t)$; then $|(u-z)(t)|=u(t)-\varepsilon y(t) \leqslant 1-\delta_{0} \varepsilon$.

4.5. Corollary. If $X \subset C(\Omega)$ is the $M$-space determined by the full set of relations $x\left(s_{\alpha}\right)=\lambda_{\alpha} x\left(t_{\alpha}\right), s_{\alpha}, t_{\alpha} \in \Omega, \lambda_{\alpha} \geqslant 0$, and if $\delta_{0}=\inf \left\{\lambda_{\alpha} ; \lambda_{\alpha} \neq 0\right\}>0$, then every bounded subset $A$ of $C(\Omega)$ has a nonempty set of Chebyshev centers in $X, Z_{X}(A)$, and a nonempty set of best compact approximants in $X, Z_{\mathcal{K}_{(X)}}(A)$, and the mappings $A \rightarrow Z_{X}(A), A \rightarrow Z_{\mathcal{K}(X)}(A)$ are Lipschitz continuous in the Hausdorff metric with constant $2 / \delta_{0}$.

Proof. The existence is immediate from Propositions 4.4 and 2.4. As to the continuity statement, given bounded sets $A, A^{\prime}$, and $z \in Z_{Y}(A)$ define $\varepsilon=$ $2 h\left(A, A^{\prime}\right) /\left(\delta_{0}\left(r_{Y}\left(A^{\prime}\right)+2 h\left(A, A^{\prime}\right)\right)\right)$. Since $r\left(z, A^{\prime}\right) \leqslant r_{Y}\left(A^{\prime}\right)+2 h\left(A, A^{\prime}\right)$, we also have

$$
r\left(z, A^{\prime}\right) \leqslant r_{Y}\left(A^{\prime}\right) /\left(1-\delta_{0} \varepsilon\right)
$$

and therefore the proof of Proposition 2.4 shows the existence of $z^{\prime} \in Z_{Y}\left(A^{\prime}\right)$ such that

$$
\left\|z-z^{\prime}\right\| \leqslant \varepsilon r\left(z, A^{\prime}\right) \leqslant 2 / \delta_{0} h\left(A, A^{\prime}\right) .
$$

This proves $2 / \delta_{0}$ to be a Lipschitz constant for the Chebyshev center map. The proof for the map $A \rightarrow Z_{\mathcal{K}(X)}(A)$ is similar.

Remark. Observe that Corollary 4.5 applies to the following particular cases:

(a) $X=C(\Omega)$ (here we have only the trivial relations $x\left(t_{\alpha}\right)=x\left(t_{\alpha}\right)$, and we have $\tilde{\delta}(\varepsilon)=\varepsilon \forall \varepsilon>0)$ - the fact that $Z(A) \neq \varnothing$ was proved first in [12].

(b) $X$ is a closed subalgebra of $C(\Omega)$ (here, by the Stone-Weierstrass theorem, the determining relations are $x(t)=x(s)$ when $t \sim s$ under the induced equivalence relation, and $x(t)=0$ for $t \in \bigcap_{x \in X} x^{-1}(0)$, and again $\left.\tilde{\delta}(\varepsilon)=\varepsilon\right)$. The fact that $Z_{X}(A) \neq \varnothing$ for bounded $A \subset X$ and that it depends continuously on $A$ was observed first in [29].

4.6. CoRollary. If $X \subset C(\Omega)$ is the $G$-space determined by the full set of relations $x\left(s_{\alpha}\right)=\mu_{\alpha} x\left(t_{\alpha}\right), s_{\alpha}, t_{\alpha} \in \Omega$, and if $\delta_{0}=\inf \left\{\left|\mu_{\alpha}\right| ; \mu_{\alpha} \neq 0\right\}>0$, then every bounded subset of $X$ has a nonempty Chebyshev center in $X$ and a best compact set approximant in $X$, and the mappings $A \rightarrow Z_{X}(A), A \rightarrow Z_{\mathcal{K}(X)}(A)$ are Lipschitz continuous in the Hausdorff metric with constant $2 / \delta_{0}$.

Proof. By [18, Theorem 3] there is a norm-1 projection from $\{x \in C(\Omega \times \Omega)$; $x\left(s_{\alpha}^{1}\right)=\mu_{\alpha} x\left(t_{\alpha}^{1}\right)$ if $\mu_{\alpha} \geqslant 0, x\left(s_{\alpha}^{2}\right)=-\mu_{\alpha} x\left(t_{\alpha}^{2}\right)$ if $\left.\mu_{\alpha}<0\right\}$ onto $X$, and we apply Proposition 2.4 and Corollary 4.5. 
Remark. Corollary 4.6 contains the particular case when $X=C_{\sigma}(\Omega)$ for some involutive homeomorphism $\sigma$ of $\Omega$ onto $\Omega$ (here the nontrivial relations are $x(t)=$ $-x(\sigma t), t \in \Omega$, and again $\tilde{\delta}(\varepsilon)=\varepsilon \forall \varepsilon>0)$.

4.7. Example. An M-space $X$ and a bounded $A \subset X$ which has no Chebyshev center and no best compact set approximant. Let

$$
\begin{aligned}
& X=\left\{x \in C[0,1] ; x\left(\frac{1}{2 n}\right)=\frac{1}{n} x\left(\frac{1}{2 n-1}\right), n=1,2, \ldots\right\}, \\
& A=\left\{x \in X ; 0 \leqslant x \leqslant \sum_{n=1}^{\infty}\left(\chi_{[1 /(2 n+1) .1 / 2 n]}+\left(1+\frac{1}{n}\right) \chi_{(1 / 2 n, 1(2 n-1))}\right)\right\} .
\end{aligned}
$$

Let $y_{n}(t)$ be the piecewise linear function with the vertices $(0,0),(1 /(2 k-1), 1)$ and $(1 / 2 k, 1 / k)$ for $k=1, \ldots, n$, and $(1 /(2 n+1), 0)$. Then $r\left(y_{n}, A\right)=1+1 /(n+1)$ $\rightarrow 1$. If $K \subset X$ is compact and $r(K, A) \leqslant 1$, take $n$ so that $y(1 /(2 n-1))<\frac{1}{2} \forall y \in K$ and then take $0<\delta<1 / 2 n$ such that $|s-t| \leqslant \delta \Rightarrow|y(s)-y(t)|<1 / 2 n \forall y \in K$. Consider the positive linear function $x$ with the vertices $(0,0),(1 / 2 n+\delta, 1+1 / n)$, $(1 /(2 n-1), 0),(1,0) . x \in A$ and therefore, for some $y_{0} \in K,\left\|x-y_{0}\right\|<1$. In particular, $y_{0}(1 / 2 n+\delta) \geqslant 1 / n$; hence $y_{0}(1 / 2 n) \geqslant 1 / 2 n$ and $y_{0}(1 /(2 n-1)) \geqslant \frac{1}{2}$, which contradicts the choice of $n$.

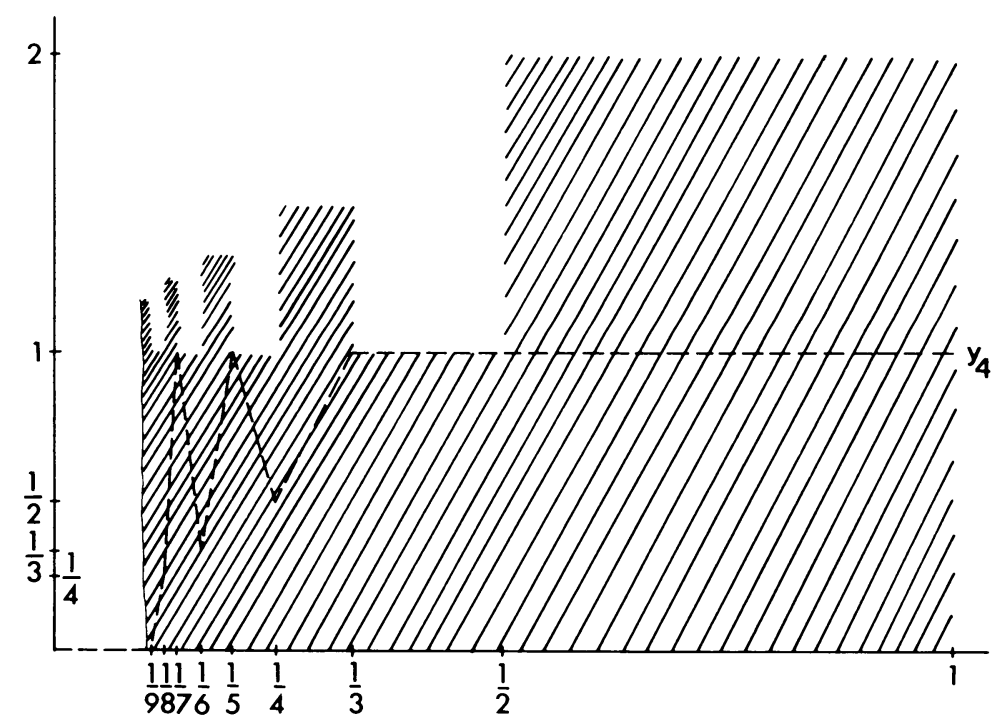

4.8. Corollary. The space $A(S)$ of continuous affine functions on a compact Choquet simplex need not have Chebyshev centers or best compact set approximants for bounded sets.

Proof. By a result of Lazar and Lindenstrauss [15, Theorem 5.5], every separable infinite-dimensional pre- $L_{1}$ Banach space is isometric to a subspace of some $A(S)$ space on which there is a norm-1 projection from $A(S)$. Thus by Example 4.7 and Proposition B, there is a set $A$ in $A(S)$ which has not best $n$-net for any $n \geqslant 1$ and no best compact set approximant. 
5. Continuous vector-valued function spaces. Observe that the quasi-uniform convexity condition for uniformly convex spaces and for $C(\Omega)$ spaces (and the more general class discussed in Proposition 4.2) can be satisfied with $z=z(x, \varepsilon, \tilde{\delta})$ depending continuously on $x$ (here $\varepsilon$ and $\tilde{\delta}$ are fixed). More precisely, a space $X$ is called continuously q.u.c. (c.q.u.c.) if for all $\varepsilon>0$ and the corresponding $\tilde{\delta}(\varepsilon)$ there is a continuous selection $z(x, \varepsilon, \tilde{\delta})$ for the point $z$ in the q.u.c. definition at the beginning of $\S 2$.

5.1. Proposition. If $X$ is c.q.u.c. then, for every topological space $\Omega, C(\Omega, X)$ is q.u.c. with $\tilde{\delta}_{C(\Omega, X)}=\tilde{\delta}_{X}$.

Proof. Given $f \in C(\Omega, X)$, let $g(t)=z(f(t), \varepsilon, \tilde{\delta}) . g \in C(\Omega, X)$ by our assumption and clearly satisfies the q.u.c. condition.

5.2. Corollary [2]. If $X$ is uniformly convex, then $C(\Omega, X)$ admits Chebyshev centers for bounded sets and the Chebyshev center map is uniformly continuous with respect to the Hausdorff metric on bounded families of sets.

If $X$ is not q.u.c., $C(\Omega, X)$ may fail to admit centers:

5.3. EXAmple. $A$ bounded subset of $c\left(L_{1}[0,1]\right)=C\left(\omega, L_{1}[0,1]\right)$ with no Chebyshev center: Let

$$
A=\left\{y \in c\left(L_{1}\right) ; y(n)=(-1)^{n} \chi_{E} /\left(1-n^{-1}\right) \mu(E), \mu(E)=(n+1) / 2 n\right\} .
$$

As in Proposition 2.8, $Z_{L_{1}}(\{y(n) ; y \in A\})=(-1)^{n} /\left(1-n^{-1}\right)$ and the radius is 1 . Let

$$
z_{m}(n)= \begin{cases}(-1)^{n} /\left(1-n^{-1}\right), & n \leqslant m, \\ 0, & n>m .\end{cases}
$$

Then $r\left(z_{m}, A\right)=1 /\left(1-m^{-1}\right) \underset{m \rightarrow \infty}{\rightarrow} 1$. Clearly, the radius 1 cannot be realized.

5.4. ExAmple. $A$ bounded subset of $c(X)=C(\omega, X), X$ 3-dimensional, without a Chebyshev center: Let $X$ be as in Example 2.5,

$$
A=\left\{y \in c(X) ; y(n) \in\left(1+n^{-2}\right)^{-1 / 2}\left[(-1)^{n} e_{3} \pm\left(e_{1}+n^{-1} e_{2}\right)\right]\right\} .
$$

As in Example 5.3, we have

$$
Z_{X}(\{y(n) ; y \in A\})=(-1)^{n}\left(1+n^{-2}\right)^{-1 / 2} e_{3}, \quad r_{X}(\{y(n) ; y \in A\})=1 .
$$

$r(0,\{y(n) ; y \in A\})=\left(1+n^{-1}\right)\left(1+n^{-2}\right)^{-1 / 2}$ shows that $r_{c(X)}(A)=1$ cannot be realized.

RemarK. Similar examples can be constructed in $c\left(l_{1}\right)$ and other $c(X)$ spaces, whenever the Chebyshev center map in $X$ is not upper semicontinuous.

\section{REFERENCES}

1. E. M.Alfsen and E. G. Effros, Structure in real Banach spaces, Ann. of Math. (2) 96 (1972), 98-173.

2. D. Amir, Cheby'shev centers and uniform convexity, Pacific J. Math. 77 (1978), 1-6.

3. D. Amir and Z. Ziegler, Relative Chebyshev centers in normed linear spaces. I, J. Approx. Theory 29 (1980), 235-252.

4. J. R. Calder, W. P. Coleman and R. L. Harris, Centers of infinite bounded sets in a normed space, Canad. J. Math. 25 (1973), 986-999. 
5. F. Deutsch, J. Mach and K. Saatkamp, Approximation by finite rank operators, J. Approx. Theory (to appear).

6. H. Fakhoury, Approximation des bornés d'un espace de Banach par des compacts et applications $\dot{a}$ l'approximation des opérateurs bornés, J. Approx. Theory 26 (1979), 79-100.

7. M. Feder, On a certain subset of $L_{1}[0,1]$ and non-existence of best approximation in some space of operators, J. Approx. Theory 29 (1980), 170-177.

8. A. L. Garkavi, The best possible net and the best possible cross section of a set in a normed space, Amer. Math. Soc. Transl. 39 (1964), 111-132.

9. The conditional Chebyshev center of a compact set of continuous functions, Math. Notes 14 (1973), 827-831.

10. A. L. Garkavi and V. N. Zamyatin, Conditional Chebyshev centers of a bounded set of continuous functions, Math. Notes 18 (1975), 622-627.

11. R. B. Holmes, M-ideals in approximation theory, Approximation Theory. II, Academic Press, New York, 1976, pp. 391-396.

12. M. I. Kadec and V. N. Zamyatin, Chebyshev centers in the space $C[a, b]$, Teor. Funkciī, Funkcional Anal. i Priložen. 7 (1968), 20-26. (Russian)

13. K. S. Lau, Approximation by continuous vector-valued functions, Studia Math. 68 (1980), 291-298.

14. __ On a sufficient condition for proximity, Trans. Amer. Math. Soc. 251 (1979), 343-356.

15. A. J. Lazar and J. Lindenstrauss, Banach spaces whose duals are $L_{1}$ spaces and their representing matrices, Acta Math. 126 (1971), 165-193.

16. A. Lima, Intersection properties of balls and subspaces in Banach spaces, Trans. Amer. Math. Soc. 227 (1977), 1-62.

17. J. Lindenstrauss, Extension of compact operators, Mem. Amer. Math. Soc. No. 65 (1964).

18. J. Lindenstrauss and D. E. Wulbert, On the classification of the Banach spaces whose duals are $L_{1}$ spaces, J. Funct. Anal. 4 (1969), 332-349.

19. J. Mach, Best simultaneous approximation of bounded functions with values in certain Banach spaces, Math. Ann. 240 (1979), 157-164.

21. _ On the existence of best simultaneous approximation, J. Approx. Theory 25 (1979), 258-265.

22.

23. J. Mach and J. D. Ward, Approximation by compact operators on certain Banach spaces, J. Approx. Theory 23 (1978), 274-286.

24. E. Michael, Selected selection theorems, Amer. Math. Monthly 63 (1956), 233-238.

25. E. Rozema and P. Smith, Global approximation with bounded coefficients, J. Approx. Theory 16 (1976), 162-174.

26. K. Saatkamp, Schnitteigenschaften und beste Approximation, Doctoral Dissertation, Bonn, 1979

27. Z. Semadeni, Banach spaces of continuous functions, Vol. 1, PWN, Warsaw, 1971.

28. P. W. Smith and J. D. Ward, Restricted centers in $C(\Omega)$, Proc. Amer. Math. Soc. 48 (1975), 165-172. 29. , Restricted centers in subalgebras of $C(X)$, J. Approx. Theory 15 (1975), 54-59.

30. J. D. Ward, Chebyshev centers in spaces of continuous functions, Pacific J. Math. 52 (1974), 283-287.

31. V. N. Zamyatin, Relative Chebyshev centers in the space of continuous functions, Soviet Math. Dokl. 14 (1973), 610-614.

School of Mathematical Sciences, Tel Aviv University, Tel Aviv, IsRael (Current address of Dan Amir)

Institut fUr Angewandte Mathematik der Universitát Bonn, D-5300 Bonn, Federal RepubLIC OF GERMANY (Current address of Jaroslav Mach)

Current address: (Klaus Saatkamp): Department of Mathematics, Texas A \& M University, College Station, Texas 77843 